\title{
THE ARGUS ELECTRON-PHOTON CALORIMETER III. Electron-hadron separation
}

\author{
A. DRESCHER, B. GRÄWE, B. HAHN, B. INGELBACH, U. MATTHIESEN, H. SCHECK, \\ J. SPENGLER and D. WEGENER
}

Institut für Physik, Universität Dortmund, Fed. Rep. Germany.

Received 2 January 1985

The high granularity of the ARGUS shower counter system allows one to determine the lateral energy deposition. An algorithm based on the number of shower counters set by an incoming particle, the amount of energy deposited in the shower counters and the lateral distribution of the energy allows to separate electrons from muons and hadrons. For electrons with momenta larger than 0.7 $\mathrm{GeV} / \mathrm{c}$ the hadron rejection rate is of the order of 50 at an electron detection efficiency of $80 \%$.

\section{Introduction}

The ARGUS spectrometer (fig. 1) is a detector for $\mathrm{e}^{+} \mathrm{e}^{-}$reactions at DORIS II [1]. It consists of a central drift chamber [2], which measures the momentum of charged particles and the rate of their energy loss, a time of flight system [3] and an electromagnetic shower detector [4]. These components are all inside the coil of the magnet, which produces a nearly homogeneous field of $0.8 \mathrm{~T}$. Muons are detected in two planes by proportional tubes [5], positioned behind absorbers, with cut off momenta of $0.7 \mathrm{GeV} / c$ and $1.1 \mathrm{GeV} / c$ respectively.

Details of the performance of the shower counters in a test beam and under experimental conditions in the ARGUS detector have been previously described [4]. In this paper we will concentrate on the question of how electrons (used as a synonym for electrons and positrons) can be separated from other charged particles hitting the shower counters. A clean electron sample, identified with high efficiency and with a small hadron admixture, is of interest for the physics program performed with the ARGUS detector, namely the study of semileptonic $B$ decays. These can be used to determine the branching ratios $\mathrm{BR}(\mathrm{b} \rightarrow \mathrm{c})$ and $\mathrm{BR}(\mathrm{b} \rightarrow \mathrm{u})$, as well as to search for $\mathrm{B}^{0}-\overline{\mathrm{B}^{0}}$ mixing. For these physics goals, the electron momentum range of main interest is $1.2 \mathrm{GeV} / c \leq p_{\mathrm{e}} \leq$ $2.5 \mathrm{GeV} / c$.

Contrary to most other experiments $[6,7]$ the separation of electrons and hadrons has to be based entirely on the differences of the lateral energy deposited by particles hitting the shower counters, because there is only one longitudinal sample. Since the lateral width of the shower counters, $r \simeq 10 \mathrm{~cm}$, is large compared with the radius of a typical electromagnetic shower (Molière radius $R_{\mathrm{M}}<5 \mathrm{~cm}$ ) but is small in comparison with the radius of a hadronic shower (absorption length $\lambda_{\mathrm{abs}} \simeq 46$ $\mathrm{cm}$ ), the two kinds of showers can be distinguished. On the other hand, minimum ionizing particles can be separated from showering electrons, simply on the basis of the energy deposited.

The paper is organized as follows: in section 2 we briefly describe those properties of the ARGUS electron photon calorimeter, which are of importance for the present analysis. Further details have been given in previous publications [4]. In section 3 the pattern of energy deposition for the different particle species is discussed. In section 4 we describe the algorithm which exploits the results of section 3 with the aim of electron hadron separation at different momenta.

\section{ARGUS electromagnetic calorimeter}

The ARGUS shower counters are of the lead scintillator sandwich type with $5 \mathrm{~mm}$ thick scintillator plates (Altustipe UV). The thickness of the lead plates is $1 \mathrm{~mm}$ in the barrel, and $1.5 \mathrm{~mm}$ in the endcap region. The overall length of each counter corresponds to 12.4 radiation lengths for electrons and 0.8 absorption lengths for pions in the momentum range of interest. The lead and scintillator plates are separated by aluminized mylar foils with $98 \%$ reflectivity. The lead scintillator sandwich is "read out" by a $3 \mathrm{~mm}$ thick wave length shifter coupled to a 1 inch photo tube (Valvo XP 2008 UB). The barrel counters consist of 64 plates of each material with $10.9 \mathrm{~cm} \times 10.3 \mathrm{~cm}$ lateral cross section. The endcap counters contain 45 plates of each material of trapezoidal shape with maximum lateral dimensions of $11.6 \mathrm{~cm}$ and $10.4 \mathrm{~cm}$.

The shower counters are mounted inside of the mag- 


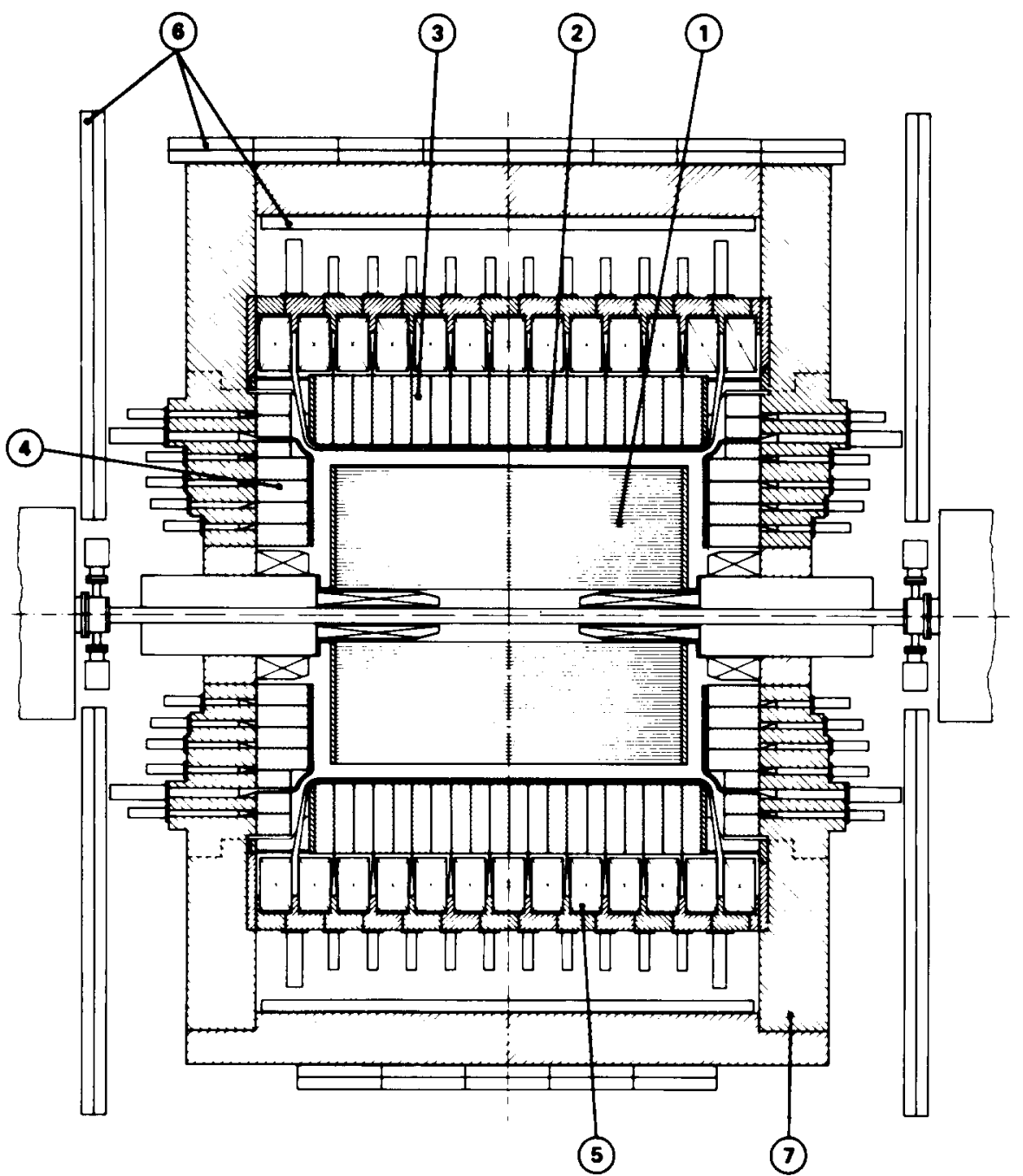

Fig. 1. Schematic view of the ARGUS detector: (1) drift chamber, (2) time of flight counters, (3) barrel shower counters, (4) end cap shower counters, (5) magnetic coil, (6) muon chambers, (7) iron yoke.

netic coil. The cylindrical arrangement of the barrel counters (fig. 1) results in a variation of the angle of incidence between $0^{\circ}$ and $45^{\circ}$ for particles coming from the interaction point. The endcap counters are arranged in a plane perpendicular to the primary beam (fig. 1), the angle of incidence for particles hitting this detector component varies between $20^{\circ}$ and $45^{\circ}$. Because of the cylindrical symmetry of the detector counters in the same polar angle region can be treated in common. Counters at $\theta=90^{\circ}$ are labelled with the ring number 1 , with decreasing polar angle the ring number increases to 15 for the counters in the endcap at the smallest polar angles.

The energy resolution presently obtained under experimental conditions is

$$
\frac{\sigma}{E}=\sqrt{\left(\frac{0.08}{\sqrt{E}}\right)^{2}+(0.068)^{2}},
$$

where $E$ is measured in $\mathrm{GeV}$.

The angular resolution is $0.7^{\circ}$ for electrons with momenta $p_{\mathrm{e}} \geq 1 \mathrm{GeV} / c$. Further details concerning the construction and properties of the shower counters are described in refs. [4b-4d].

\section{Pattern of energy deposition for different particle species}

The following information about the energy deposited by a particle in the ARGUS shower counters is 
available: the total visible energy deposited, $E$, the number of neighbouring counters hit (cluster size. $n$ ) and the visible energy $E_{i}$ detected in each counter of a cluster:

$E=\sum_{1}^{n} E_{i}, \quad E_{1}>E_{2}>\cdots>E_{n}$.

For electrons, muons and interacting and noninteracting hadrons, these measurable quantities differ in their mean and their distribution, therefore they can be used to separate different kinds of particles. Moreover the values obtained depend on the momentum of the particle. Since the ARGUS shower detector has cylindrical symmetry, the angle of incidence for a particle varies with its polar angle with respect to the beam axis (fig. 1), hence $E, E_{i}$ and $n$ show in addition a dependence on the polar angle $\theta$.

These relationships have been determined for the different particle species using a data set collected with the ARGUS detector. A clean sample of electrons at different energies is available from the reactions

$\mathrm{e}^{+} \mathrm{e}^{-} \rightarrow \mathrm{e}^{+} \mathrm{e}^{-}$.

$\mathrm{e}^{+} \mathrm{e}^{-} \rightarrow \mathrm{e}^{+} \mathrm{e}^{-} \gamma$.

The electron momentum was measured in the drift chamber. A sample of muons of varying momenta was available from the processes

$\mathrm{e}^{+} \mathrm{e}^{-} \rightarrow \mu^{+} \mu^{-}$,

$\mathrm{e}^{+} \mathrm{e}^{-} \rightarrow \mu^{+} \mu^{-} \gamma$.

The muons have been identified with the muon chambers [5]. The sample of hadron tracks was taken from the data collected on the $r$-resonance, since the admixture of leptons for this data set is smallest. To suppress leptonic decay channels, events were used with more than ten neutral and charged particles. A small admixture of leptons due to weak decays of the hadrons is unavoidable for this data set, therefore the values for the separation power given in this paper are lower limits. For hadron momenta smaller than $0.7 \mathrm{GeV} / c, \pi$ and $\mathrm{K}$-mesons can be separated with the time of flight and the $\mathrm{d} E / \mathrm{d} x$ systems, while protons and antiprotons are identified up to momenta of $1.2 \mathrm{GeV} / \mathrm{c}$ in the same manner.

\subsection{Energy deposited by electrons in the shower counters}

Since electrons deposit their total energy in the calorimeter, the electron momentum, as measured in the drift chamber, is a linear function of the energy detected by the shower counters (fig. 2). The width of the distribution is determined by the resolution of the shower counters $\sigma_{E}$ and the momentum resolution of the drift chamber, which has been measured to be

$\sigma_{p} / p=0.012 p, \quad(p>1 \mathrm{GeV} / c)$.

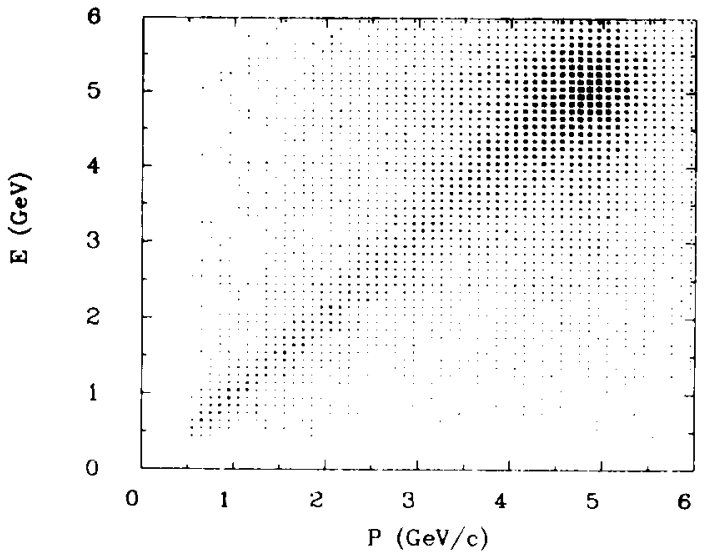

Fig. 2. Energy $E$ deposited in the shower counters vs momentum for electrons.

Adding these two contributions to the resolution quadratically

$\sigma^{2}=\sigma_{F}^{2}+\sigma_{E}^{2}$,

a cut

$|E-p c|<N \sigma, \quad N=3,4, \ldots$

can be applied.

The dependence of the electron detection efficiency as a function of $N$ is shown in fig. 3. For $N=3$ one obtains a detection efficiency of $88 \%$. This is smaller than the expected value for a normal distribution because the two distribution functions are only approximately Gaussian. The detection efficiency for a given $N$ is approximately independent of momentum [8].

Since the electromagnetic shower has a finite spatial width and the particles in general traverse more than one shower counter, the energy deposited is spread over a cluster of neighbouring shower counters. In the analysis, only counters with an energy deposit larger than 10

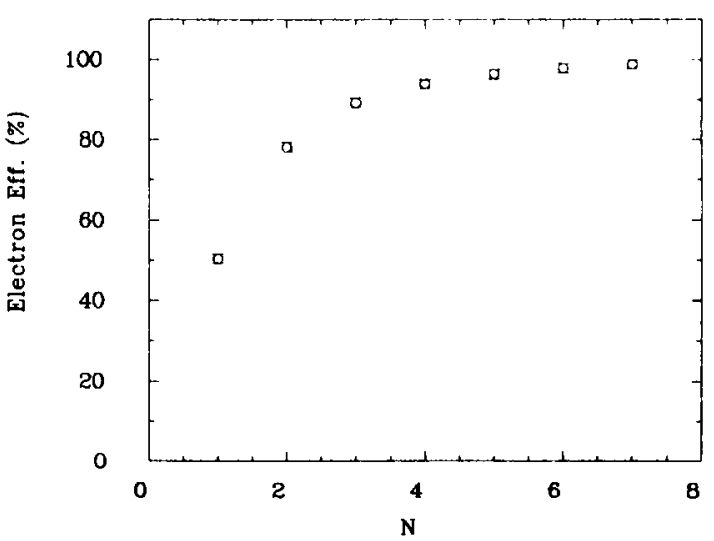

Fig. 3. Percentage of electrons passing the cut $|E-p|<N \sigma$ as a function of $N$. 
$\mathrm{MeV}$ are taken into account to suppress background. For a given type of particle the mean number of shower counters hit (cluster size $n$ ) depends on the particle momentum and the polar angle of the incoming particle $n=n(p, \theta)$.
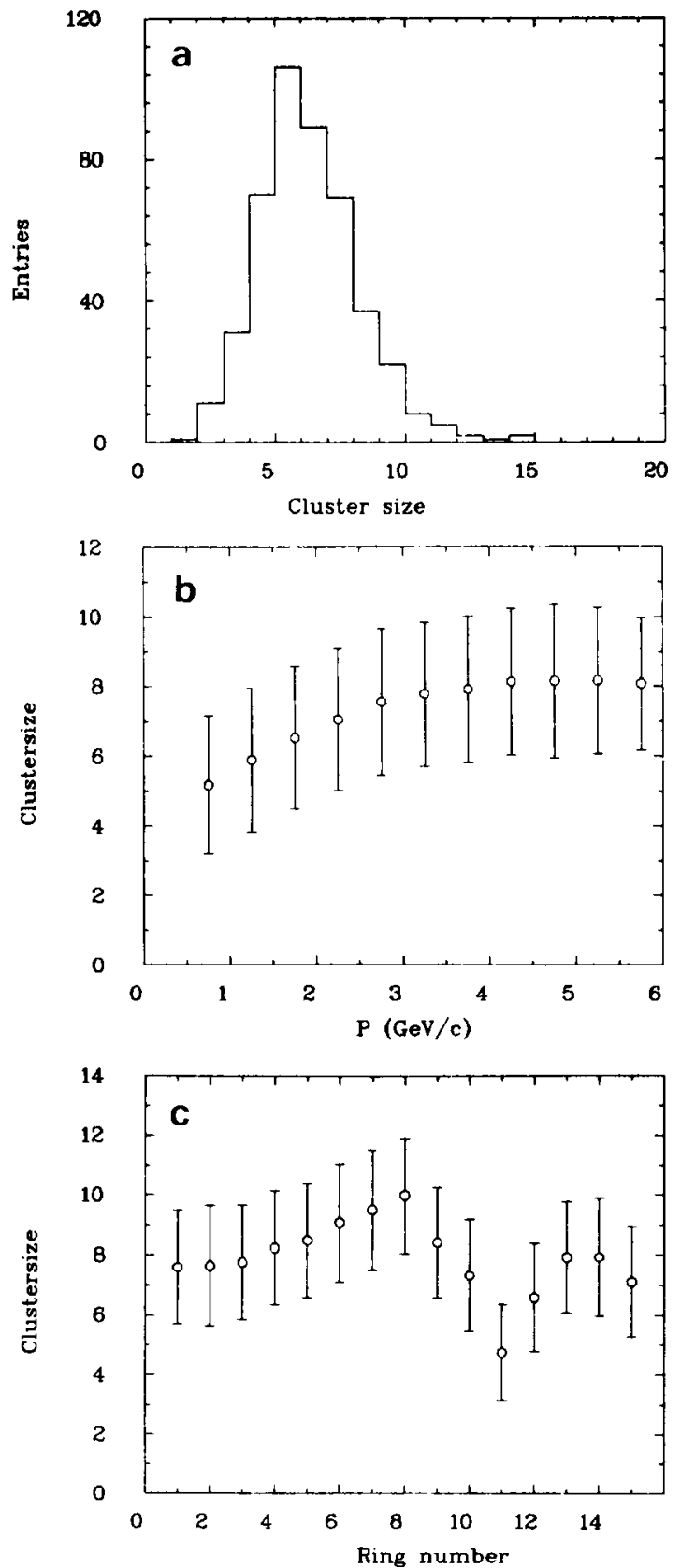

Fig. 4. (a) Cluster size distribution for electrons $(1 \mathrm{GeV} / c \leq p$ $\leq 2 \mathrm{GeV} / c$ ) hitting the shower counters. (b) Mean value of the cluster size vs electron momentum, The error bars indicate the width of the cluster size distribution. (c) Mean cluster size as a function of the ring number, which is a measure of the polar angle of the particle.
A typical distribution of cluster size for the electron momentum interval of interest is shown in fig. 4a. The distribution has a large width due to the averaging over many impact directions and the inherent shower fluctuations. The mean cluster size increases linearly
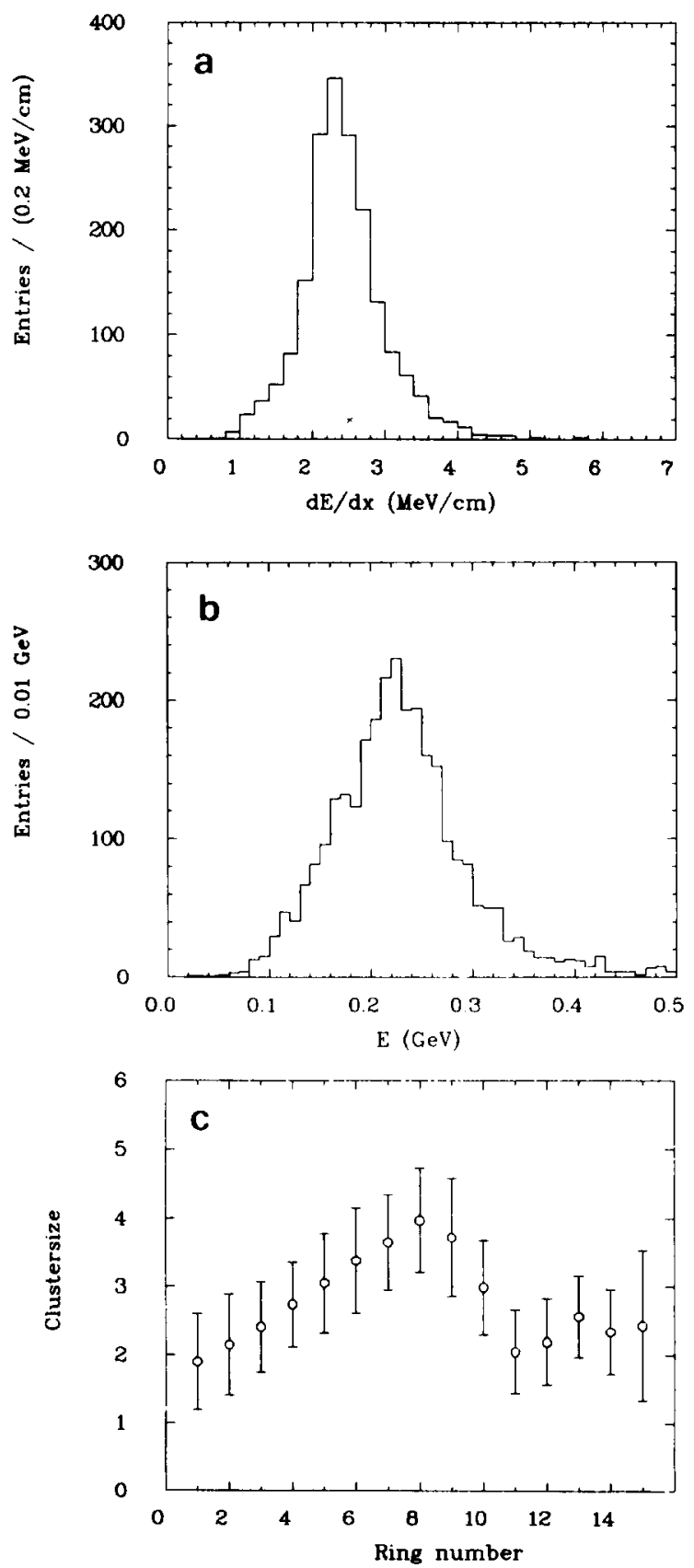

Fig. 5. (a) Energy loss of muons per unit track length in the shower counters. (b) Total energy loss of muons in the shower counters. (c) Cluster size and width of muons, hitting the shower counters as a function of the ring number. 
with the electron momentum, while its width is approximately constant (fig. $4 \mathrm{~b}$ ). The dependence of the cluster size on the polar angle is shown in fig. $4 \mathrm{c}$ for electrons with high momentum. The cluster size increases weakly with decreasing polar angle (fig. $4 \mathrm{c}$ ). The decrease observed at polar angles of $\theta \approx 45^{\circ}$ (ring 10 , 11 ) is due to the gap between the barrel and the end cap counters (fig. 1).

\subsection{Energy deposition by muons in the shower counter}

Muons lose energy in the shower counters only by ionization and excitation processes. Normalizing the visible energy in the shower counters to the track length in the scintillator, one gets (fig. 5a) a mean energy loss per unit length of

$\langle\mathrm{d} E / \mathrm{d} x\rangle=2.4 \mathrm{MeV} / \mathrm{cm}$,

in good agreement with the expected value. The total energy deposited by muons in the shower counters is
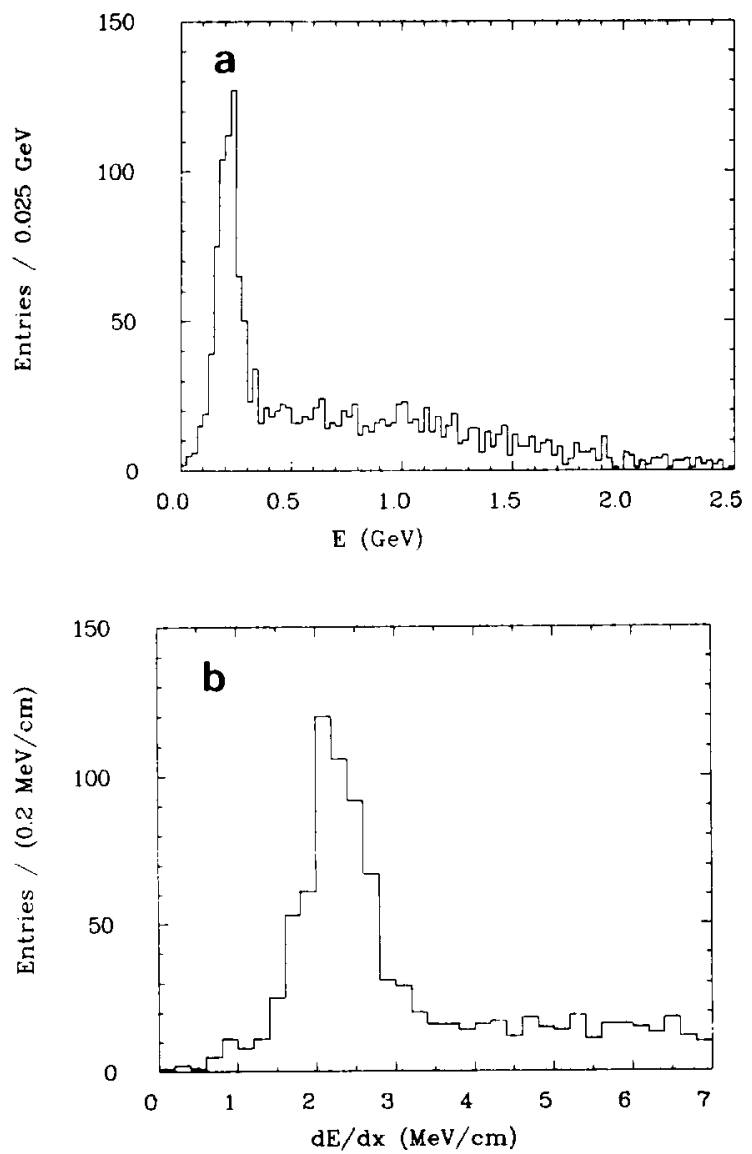

Fig. 6. (a) Energy deposited by hadrons in the shower counters. (b) Energy per unit track length deposited in the shower counters. less than $500 \mathrm{MeV}$ (fig. $5 \mathrm{~b}$ ). The cluster size as a function of the polar angle has a shape (fig. $5 \mathrm{c}$ ) similar to the one for electrons, but the mean number of counters hit is smaller than 4 and therefore differs appreciably from the cluster size for electrons (fig. 4c).

From these observations it follows that a cut on the visible energy and on the cluster size is a powerful means of distinguishing between muons and electrons.

\subsection{Energy deposition of hadrons in the shower counters}

The energy deposited by hadrons in the shower counters is due to two processes. Noninteracting hadrons lose their energy by ionization and excitation. Hadrons that interact strongly initiate a hadron shower, whose energy is partially deposited in the shower counters. These two components can be clearly separated for particle momenta $p \geqq 2 \mathrm{GeV} / c$ (fig. 6a). The visible energy in the shower counters has a pronounced peak at the position expected for minimum ionizing particles
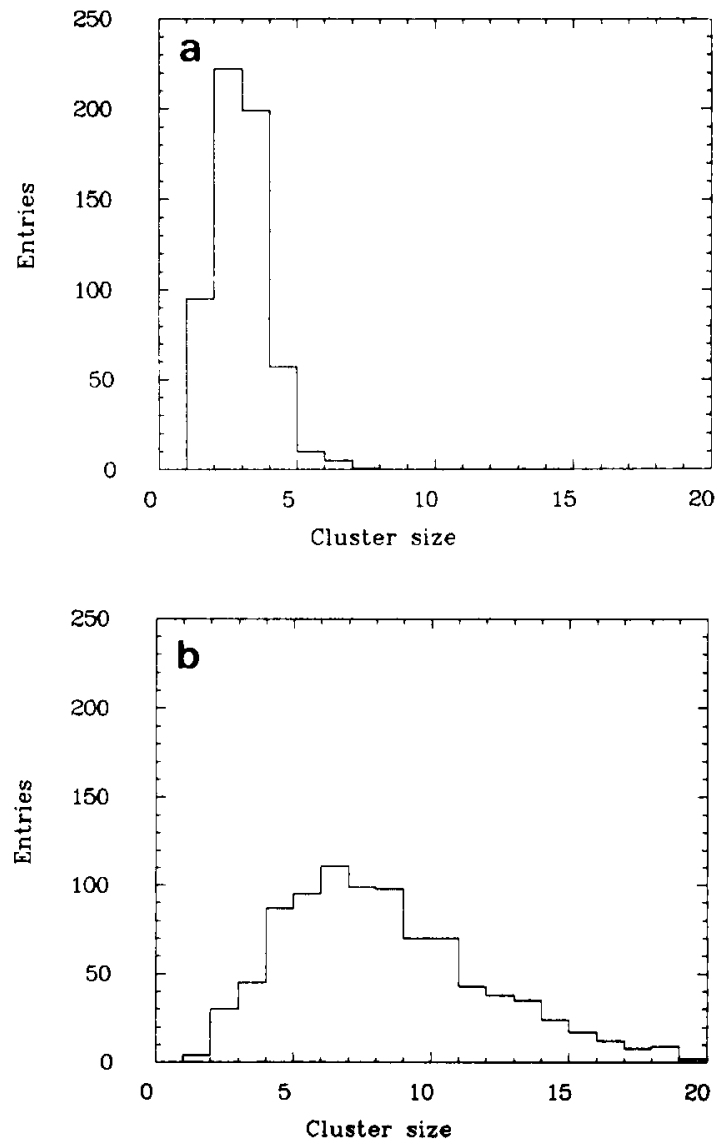

Fig. 7. (a) Cluster size distribution of noninteracting hadrons in the shower counters. (b) Cluster size distribution of interacting hadrons in the shower counters. 
(fig. 6b). In addition, a broad continuum extends to high energies which is due to interacting hadrons. Cutting at an energy of $0.35 \mathrm{GeV}$ approximately separates these two components. The measured cluster size of these two components shows the expected behaviour for minimum ionizing particles (fig. 7a) and interacting
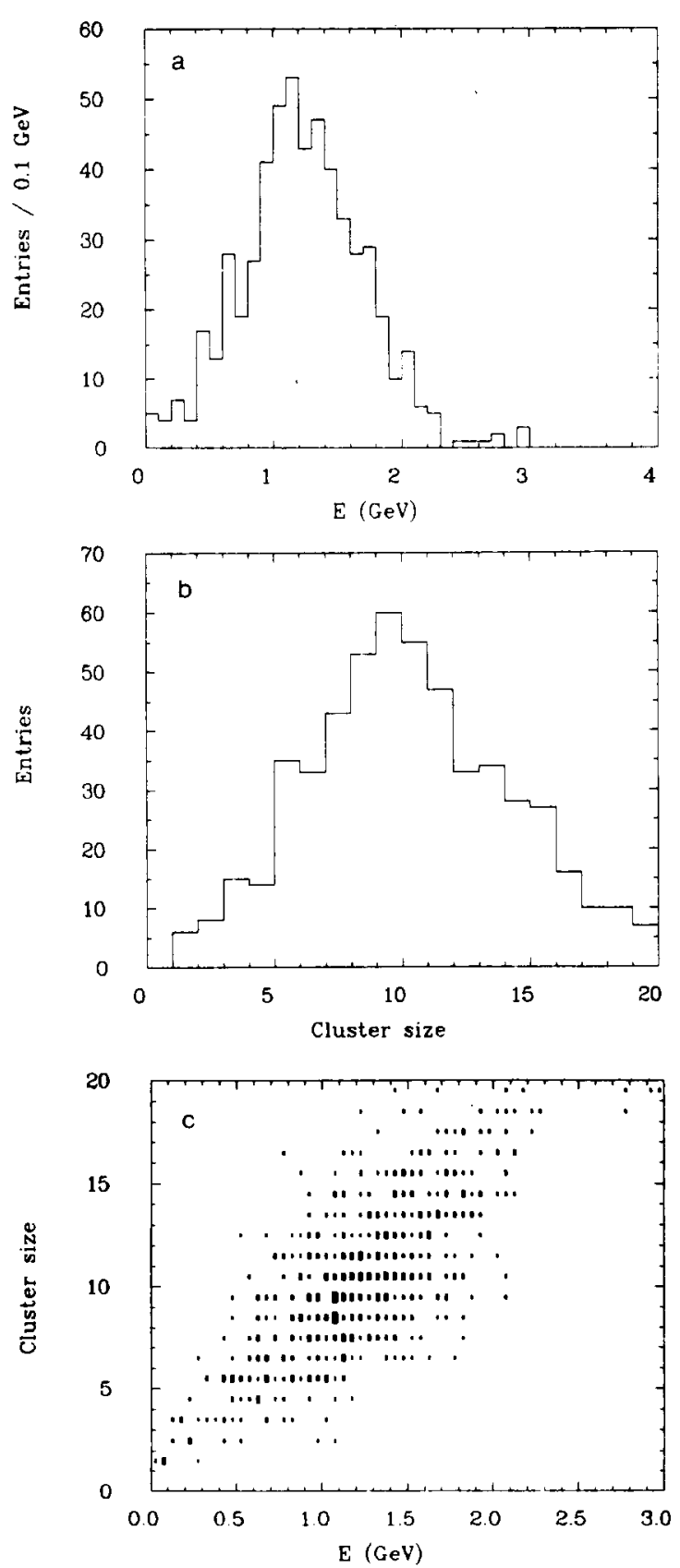

Fig. 8. (a) Energy deposited by antiprotons in the shower counters. (b) Cluster size distribution of antiprotons in the shower counters. (c) Cluster size vs energy distribution for antiprotons in the shower counters. hadrons (fig. 7b) respectively. Moreover the distribution of the cluster size as a function of the polar angle for interacting hadrons is flat, while the dependence for noninteracting hadrons coincides with that measured for muons (fig. $5 \mathrm{c}$ ). The fraction of interacting hadrons derived from figs. 6 and 7 is $(60.5 \pm 2.0) \%$ and $(50.4 \pm$ $1.9) \%$ respectively; it is in agreement with the expected fraction of interacting hadrons derived from the interaction length of pions. The difference between the two results shows that cuts on the cluster size and the energy deposition do not result in clean samples of interacting and noninteracting hadrons.

Antiprotons in the momentum range of interest behave differently. Due to annihilation processes, they deposit an appreciable amount of their total energy in the shower counters. For antiprotons with $p<1 \mathrm{GeV} / c$, the energy deposition and the cluster size are plotted in figs. $8 \mathrm{a}, \mathrm{b}$. Comparing figs. $8 \mathrm{a}, \mathrm{b}$ and figs. $6 \mathrm{a}, 7 \mathrm{~b}$ one observes a greater energy deposition and cluster size for the antiproton sample than for interacting hadrons. The cluster size depends strongly on the amount of energy deposited in the shower counters (fig. 8c). It should be noted that this dependence is steeper than for electrons. These observations can be exploited to enhance the fraction of antiprotons in the hadron sample.

\section{Particle separation with shower counters}

In the previous section we have demonstrated that the energy deposition of different particle species shows distinct features. They can be used to separate electrons from hadrons and muons. Since hadrons lose their energy in the shower counter by two processes, the separation procedure has to handle these classes separately. The energy deposition of noninteracting hadrons agrees with that of muons, therefore the algorithm applied to this class of hadrons can best be demonstrated by comparing the energy loss of muons and electrons in the shower counters. The separation of muons from electrons is based mainly on the differences in the visible energy detected in the shower counters. Interacting hadrons, on the other hand, initiate a hadronic shower which may deposit an amount of energy comparable to that of an electron with equal momentum. Therefore in this case more sophisticated methods, have to be applied.

\subsection{Electron-muon separation}

The separation of electrons from muons and non-interacting hadrons is based on two observations. In fig. $5 \mathrm{~b}$ it was shown that the energy loss of muons with $p>1 \mathrm{GeV} / c$ is always smaller than $0.5 \mathrm{GeV}$, while electrons with these momenta are only accepted if their energy deposition is larger than $0.5 \mathrm{GeV}$. Furthermore 
the cluster-size for electrons and muons differs strongly (figs. $4 \mathrm{c}, 5 \mathrm{c}$ ). Cutting on the cluster size therefore allows one to detect electrons with high efficiency and to reject all muons. For particles with $p>1 \mathrm{GeV} / c$ a cut on the energy deposition $(E>0.5 \mathrm{GeV} / c)$ and on the cluster size $(n>3)$ ensures a separation of electrons from minimal ionizing particles of over 500. The separation is defined as the probability of electrons passing the applied cuts to that of muons.

\subsection{Separation of electrons from interacting hadrons}

The separation of showers induced by electrons from those induced by hadrons is based on two experimental observations. For electromagnetic showers the deposited energy $E$ and the particle momentum $p$ are strongly correlated (fig. 8c), while for hadrons no correlation is observed (fig. 9). A cut

$|E-p c|<N \sigma$

( $N=3$ or 4 ) already rejects an appreciable fraction of the hadrons while maintaining a detection efficiency (fig. 3) for electrons of better than $88 \%$. This procedure has also been applied in other experiments [6,7]. The rejection power depends on the resolution of the detector, with the best values having been attained with a BGO shower detector [6e].

In a second step we exploit the difference in the lateral energy spread of the showers initiated by electrons and hadrons. The major fraction of energy for an electromagnetic shower is concentrated in a cone with a radius of a few millimeters, therefore $90 \%$ or more of the energy of an electron shower is deposited in the counter hit by the incoming particle (fig. 10, open circles), and only a small fraction of the total energy is shared by the neighbouring counters. Hadron induced showers (fig. 10, full circles) differ drastically from this

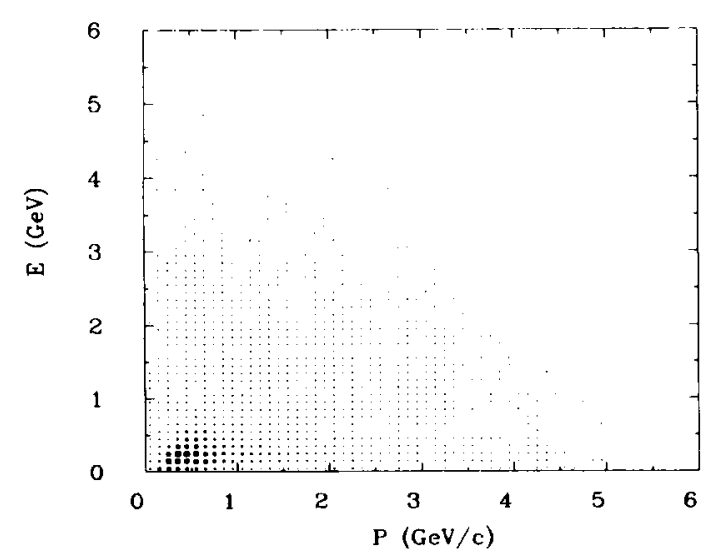

Fig. 9. Energy deposited by hadrons in the shower counters vs momentum.

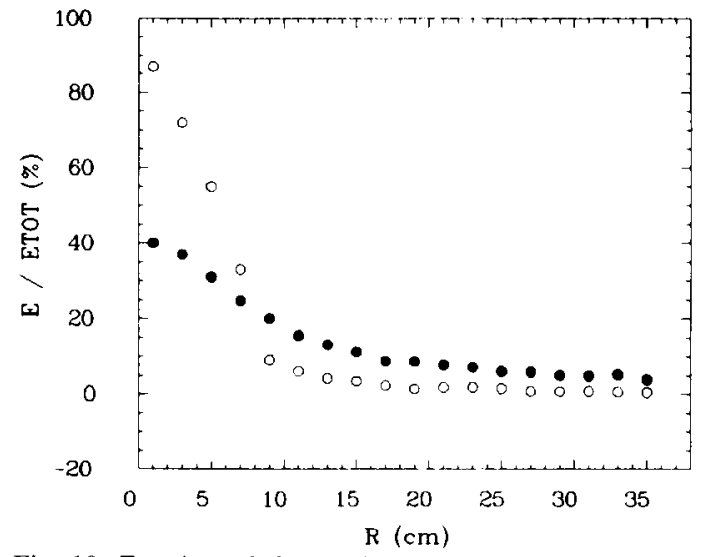

Fig. 10. Fraction of the total shower energy deposited in the distance $R$ from the impact point for electrons (open circles) and hadrons (full circles) depositing $E \geq 0.6 \mathrm{GeV}$ in the calorimeter.

behaviour. The deposited energy is shared by many neighbouring counters.

Starting from this observation we have developped an algorithm which allows one to separate electrons from interacting hadrons. To this end the lateral energy distribution for all showers with a cluster size $n \geq 3$ and an energy deposition $E \geq 0.6 \mathrm{GeV} / c$ is characterized by

$\boldsymbol{\sigma}_{r}^{2}=\sum_{i=3}^{n} \frac{\left(\boldsymbol{r}_{i}-\boldsymbol{r}\right)^{2} E_{i}}{\langle\boldsymbol{r}\rangle^{2}}$

where

$\boldsymbol{r}_{i}=$ radius vector of the counter $i$,

$E_{i}=$ energy deposition in counter $i$ with $E_{1} \geq E_{2} \geq$ $\cdots \geq E_{n}$

$\langle r\rangle \approx 10 \mathrm{~cm}$, average distance between the centers of two counters, and

$r=\frac{\sum_{1}^{n} E_{i} \boldsymbol{r}_{i}}{\sum_{1}^{n} E_{i}}$,

the radius vector of the cluster.

Note that the two counters with the highest energy deposition do not contribute to $\sigma_{r}$. The fractional width is defined by

$f_{\mathrm{LAT}}=\frac{\boldsymbol{\sigma}_{r}^{2}}{\boldsymbol{\sigma}_{r}^{2}+E_{1}+E_{2}}$,

with $0 \leq f_{\text {lat }} \leq 1$. This variable can be used to separate electrons from hadrons as demonstrated in figs. $11 \mathrm{a}, \mathrm{b}$, where for electrons and hadrons respectively $f_{\text {lat }}$ is plotted as a function of the energy deposited in the shower counters. A clear difference between the two distributions exists for $E \geq 0.6 \mathrm{GeV}$. This is demonstrated in more detail in figs. $12 \mathrm{a}-\mathrm{c}$, where for three 

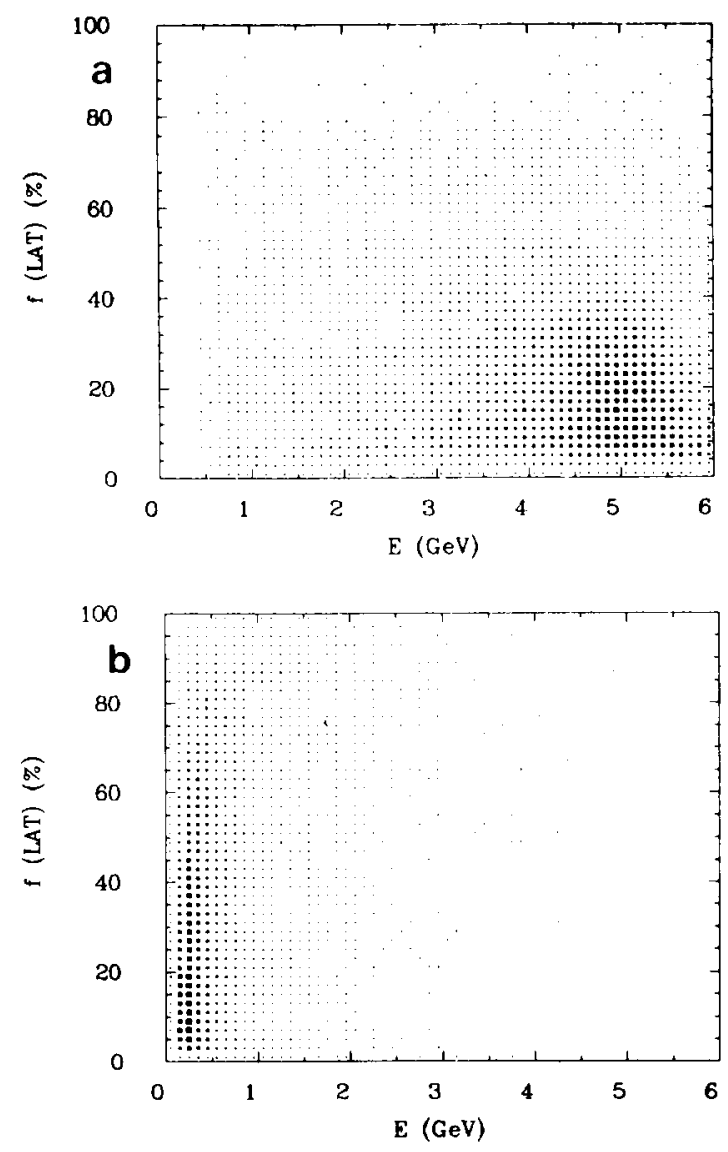

Fig. 11. $f_{\text {lat }}$ parameter defined in the text as a function of the energy $E$ deposited in the shower counters for (a) electrons and (b) hadrons.

energy regions the $f_{\text {lat }}$ distributions for electrons (solid line) and hadrons (dotted line) are plotted. The distributions are normalized to the same area. The electronhadron separation improves strongly with increasing momentum of the particles hitting the shower counters.

Different sets of cuts were applied to the data and their influence on the percentage of electrons and hadrons passing these cuts were studied (fig. 13). The results for the following three sets of cuts are especially instructive:

Set I: cluster size $n \geq 4$ and $|E-p|<3 \sigma$ (open triangles).

Set II: Set $\mathrm{I}$ of cuts and $E \geq 0.6 \mathrm{GeV}, f_{\text {lat }} \leq a$ (ring number), where $a$ is adjusted for each ring (fig. 1) in such a way that $5 \%$ of all electrons are rejected for the ring due to these cuts (full squares).

Set III: Set I of cuts and $E \geq 1 \mathrm{GeV}, f_{\text {lat }} \leq b$ (ring number), where $b$ is adjusted such that $10 \%$ of all electrons are rejected for the corresponding ring due to these cuts (open circles).
The separate treatment of each ring is necessary since not only the cluster size (figs. $4 \mathrm{c}, 5 \mathrm{c}$ ) but also the $f_{\text {lat }}$ distribution varies from one ring to another. To take this fact into account the detection efficiency of electrons (fig. 13a) and hadrons (fig. 13b) and the ratio of these efficiencies (fig. 14a) (separation) was calculated by weighting the contribution of each ring in such a way
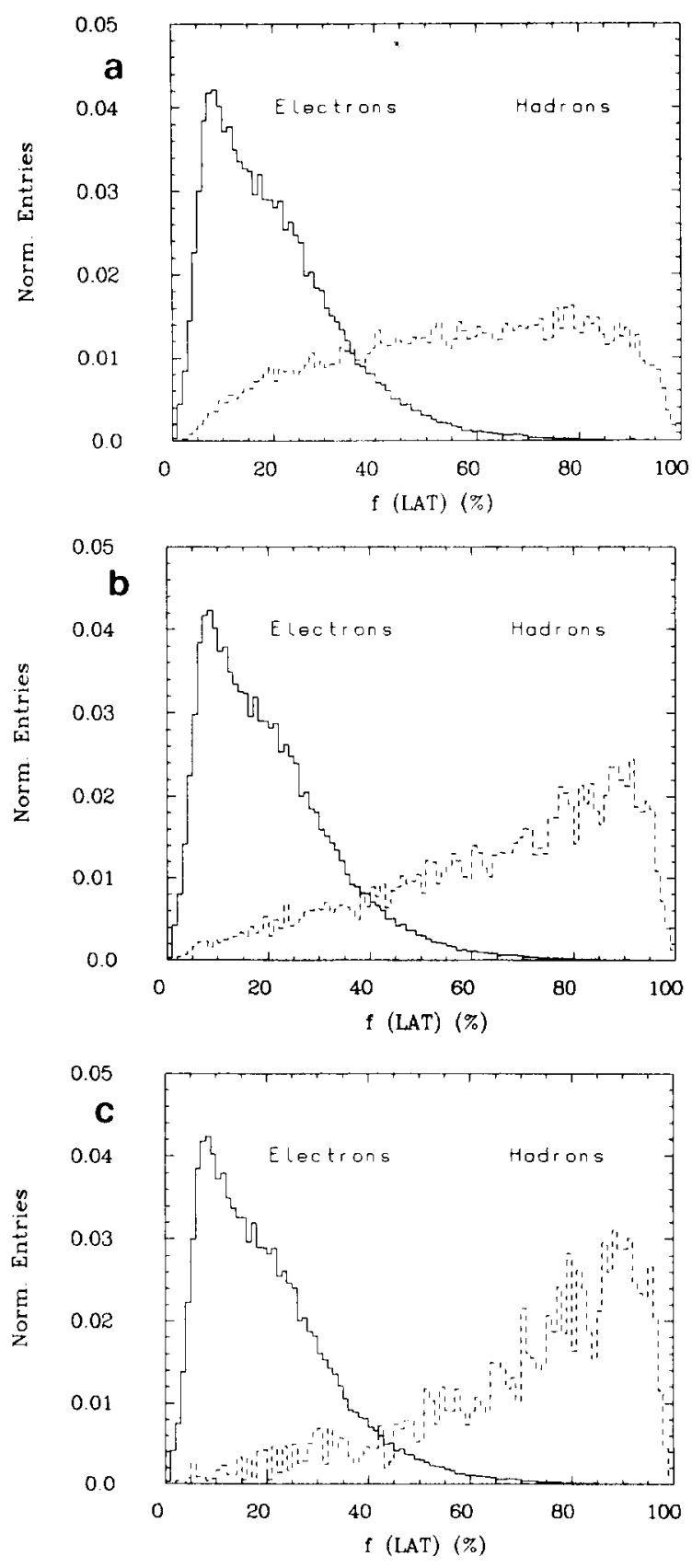

Fig. 12. Distribution of $f_{\text {lat }}$ for electrons (full line) and hadrons (broken line) for three energy intervals (a) $E \geq 0.6 \mathrm{GeV}$, (b) $E \geq 1.0 \mathrm{GeV}$, (c) $E \geq 1.5 \mathrm{GeV}$. 

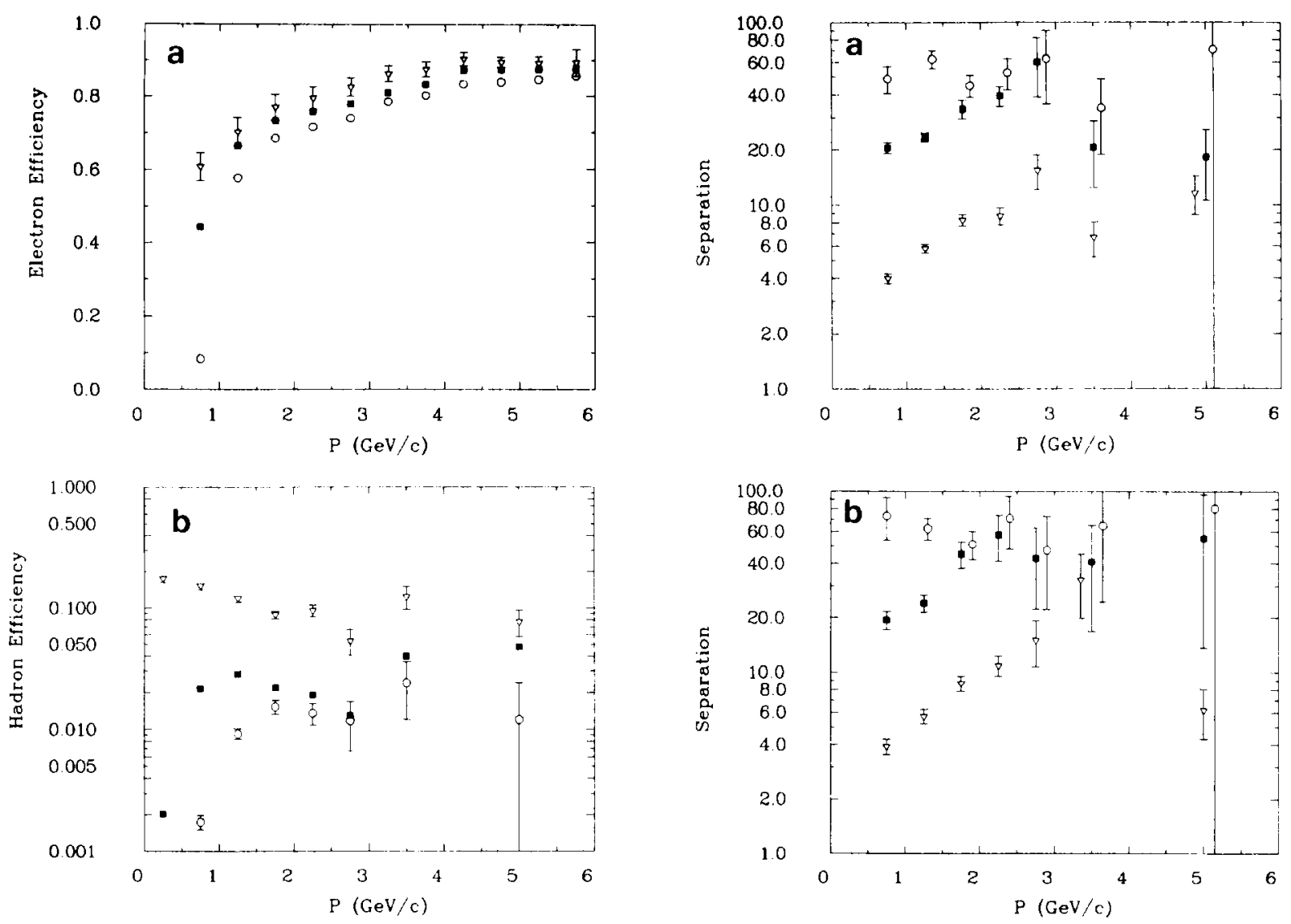

Fig. 13. (a) Electron detection efficiency and (b) hadron detection efficiency as a function of the particle momentum for the full detector. $\nabla$ Set of cuts I (see text). a Set of cuts II (see text). $O$ Set of cuts III (see text).

that equal numbers of tracks from each ring are considered when calculating the respective variable.

The cut on the lateral shape of the energy distribu-

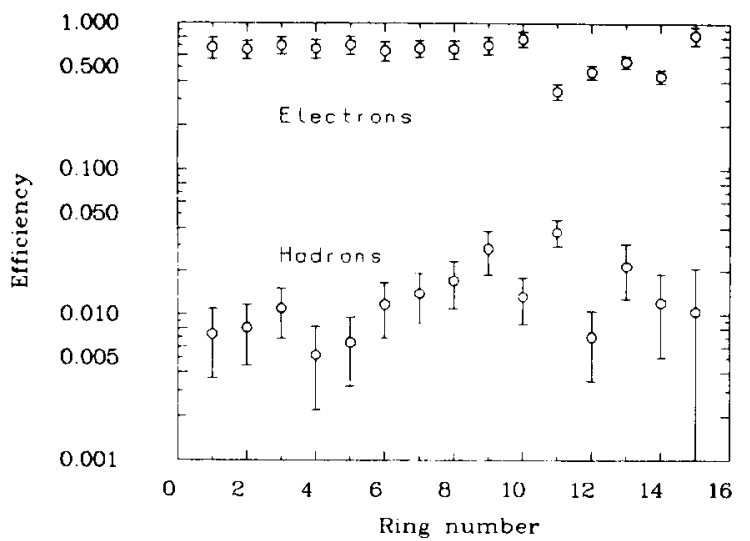

Fig. 15. Electron and hadron detection efficiency for particles with momentum $1.5 \mathrm{GeV} / c \leq p \leq 2.5 \mathrm{GeV} / c$ and the set of cuts III as a function of the ring number.

Fig. 14. Separation = electron detection efficiency/hadron detection efficiency for different particle momenta: (a) full shower detector, (b) barrel shower counters (fig. 1). The symbols are defined in fig. 13.

tion improves the separation by more than a factor 5 (fig. 14a), while the separation for the set of cuts II and III respectively are equal within the error limits for the

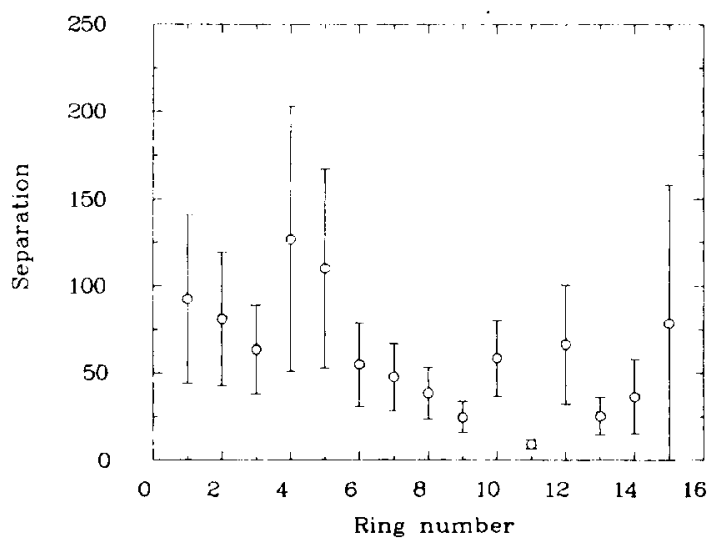

Fig. 16. Separation of electrons and hadrons for particles with momentum $1.5 \mathrm{GeV} / c \leq p \leq 2.5 \mathrm{GeV} / c$ as a function of the ring number. 


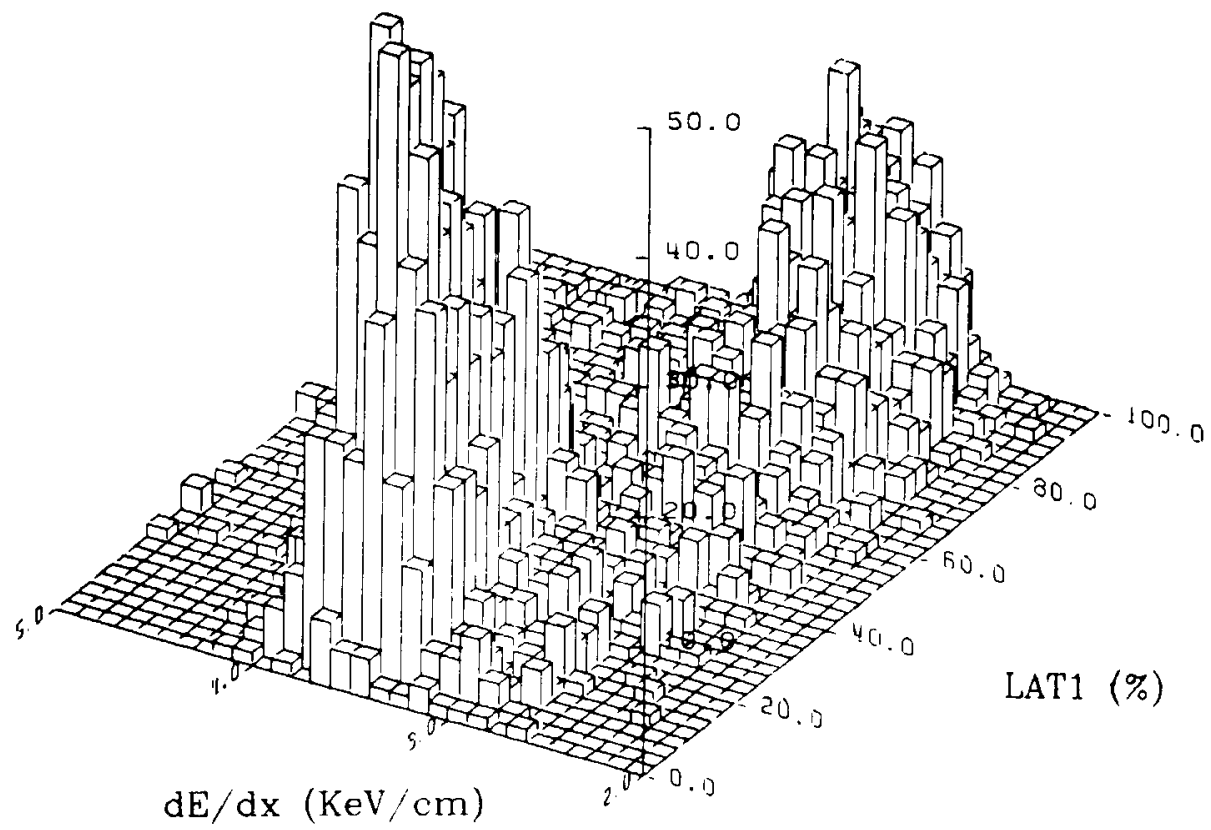

Fig. 17. $f_{\text {lat }}$ vs $\mathrm{d} E / \mathrm{d} x$ in the drift chamber for hadrons (right peak) and electrons (left peak) with momentum $1.5 \mathrm{GeV} / c \leq p \leq 2.5$ $\mathrm{GeV} / c$.

most interesting momentum interval $p \geq 1.5 \mathrm{GeV} / c$. The results for sets II and III differ for low momentum electrons $(p \leq 1.5 \mathrm{GeV} / c)$. For the most interesting momentum interval for the experiment the separation of electrons and hadrons is larger than 50, while the electron detection efficiency exceeds $70 \%$. The results for the barrel part of the shower counters (fig. 1) are given separately in fig. $14 \mathrm{~b}$, the separation of electrons and hadrons is better than for the full detector. For momenta $p \geq 1.5 \mathrm{GeV} / c$ the electron and hadron efficiency are given separately in fig. 15 while the resulting separation is shown in fig. 16 as a function of the ring number. These plots demonstrate clearly the influence of the ring number on the separation. Thus a further improvement by a factor of 2 in the separation of electrons from hadrons can be achieved if one restricts the polar angle to the barrel region. This may be of interest if a very clean electron sample is needed.

The electron hadron separation attainable with the full ARGUS detector can be improved beyond the limits determined by the shower counters if one uses the energy loss of charged particles in the drift chamber [2]. In the drift chamber, electrons lose more energy than minimum ionizing particles (fig. 17). A combined cut on the shower counter information and the $\mathrm{d} E / \mathrm{d} x$ signal as measured in the drift chamber results in an electron-hadron separation larger than 250 .

\section{Summary}

The lateral shower energy deposition in the ARGUS electromagnetic calorimeter has been shown to have characteristic features which differ for electrons and hadrons. An algorithm has been developped which rejects hadrons with high probability $(>50)$, while the electron detection efficiency is still larger than $70 \%$. The electron hadron separation power increases with the particle momentum.

The algorithm developed to separate electrons and hadrons on the basis of their lateral shower deposition turns out to be nearly as efficient as the one making use of the different longitudinal shower development of hadrons and electrons $[6,7]$.

We acknowledge the help from our colleagues of the ARGUS collaboration whose cooperation made this analysis possible. We thank the DESY directorate for the support and the kind hospitality extended to us. This work was supported by the Bundesministerium für Forschung und Technologie of the Federal Republic of Germany.

\section{References}

[1] ARGUS - A new detector for DORIS, DESY F15/PRO148/1978.

[2] M. Danilov et al., Nucl. Instr. and Meth. 217 (1983) 153. 
[3] R. Heller et al., The ARGUS time of flight system, IHEPHD/84-03, submitted to Nucl. Instr. and Meth.

[4] (a) W. Hofmann et al.. Nucl. Instr. and Meth. 195 (1982) 475; (b) A. Drescher et al., Nucl. Instr. and Meth. 205 (1983) 125; (c) A. Drescher et al.. Nucl. Instr. and Meth. 216 (1983) 35; (d) J. Spengler. Proc. 3rd Int. Conf. on Instrumentation for Colliding Beam Physics, Novosibirsk (1984) p. 81.

[5] A. Arefiev et al., The ARGUS muon chambers DESY 83-025

[6] (a) L.M. Ledermann et al., Nucl. Instr. and Meth. 129 (1975) 65; (b) E. Gabathuler et al., Nucl. Instr. and Meth. 157 (1978) 47; (c) J.H. Cobb et al.. Nucl. Instr. and Meth.
158 (1979) 93; (d) A. Babaev et al. Nucl. Instr. and Meth. 160 (1978) 427; (e) H. Dietl et al., MPI-PAE/EXP. El. 124 (1983); (f) B. Cox et al., A measurement of the response of an SCG1-C Scintillation glass array to $4-14 \mathrm{GeV} / \mathrm{c}$ pions, FNAL (1984).

[7] (a) J.A. Appel et al., Nucl. Instr. and Meth. 127 (1975) 495: (b) E. Locci et al., Nucl. Instr. and Meth. 164 (1979) 97; (c) G. Battistoni, Proc. Int. Conf. on Instrumentation for Colliding Beam Physics (1982) p. 195; (d) B.M. Bleichert et al., Particle Identification in an electron/hadron calorimeter Si-82-13.

[8] B. Hahn. Diplomarbeit, Dortmund (1984). 